\title{
Divulgatio
}

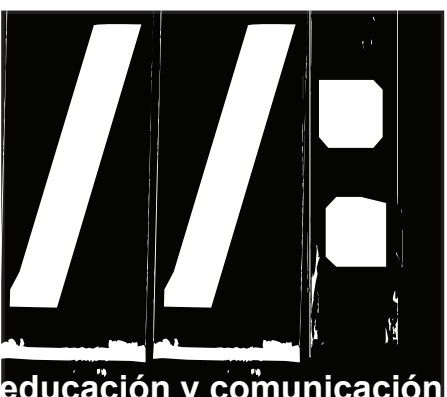

9: 117-135 Nov. 2014

\section{EXPERIENCIA DE UN DIAGNÓSTICO SOBRE LA SITUACIÓN ACTUAL DEL INSTITUTO SUPERIOR POLITÉCNICO DE KWANZA SUL CANGOLA] EN LA GESTIÓN DEL CONOCIMIENTO CON EMPLEO DE LAS TIC DIRIGIDO AL PROGESO DE ENSEÑANZA APRENDIZAJE Experience of diagnosis on the current situation of the Polytechnic Superior Institute of Kwanza Sul [Angola] in knowledge management with the use of ICT in the teaching-learning process}

Ing. Augusto Da Silva Sachonga.

Profesor de Informática del Instituto Superior Politécnico de Kwanza Sul. Jefe Técnico de la Empresa de Telecomunicaciones de Angola

"Angola Telecom". (República de Angola).

E.mail:silpedroa@hotmail.com

Dr. C. Alexander López Padrón. Profesor Titular. Director del Centro de Estudios de la Educación Superior Agropecuaria de la Universidad Agraria de La Habana. (Cuba).

E.mail: alejo@unah.edu.cu

Dr. C. Alexis Torres Alonso. Profesor Titular. Decano de la Facultad de Ciencias Pedagógicas de la Universidad Agraria de La Habana. (Cuba) E.mail: alexist@unah.edu.cu

Resumen:

En el presente artículo se detalla el estudio descriptivo diagnóstico de la situación actual de la gestión del conocimiento con empleo de las TIC dirigido al proceso de enseñanza aprendizaje de pregrado en el Instituto Superior Politécnico de Kwanza Sul (Angola). Como herramienta de recogida de datos se emplearon los cuestionarios validados por Iriarte (2008) "Valoración del proceso de gestión de información destinada a la producción de contenidos dirigidos al proceso de enseñanza aprendizaje por profesores universitarios" y "Valoración del proceso de gestión de información destinada a la actividad de estudio en el proceso de enseñanza aprendizaje por estudiantes universitarios". Los resultados obtenidos muestran un bajo nivel de utilización de las TIC, baja disponibilidad de recursos tecnológicos para la gestión del conocimiento, uso limitado y poco preciso de la variedad de herramientas y servicios existentes para la producción y gestión de materiales educativos, así como una inadecuada alfabetización informacional.

Palabras claves: gestión del conocimiento, formación, instituciones de educación superior

\section{Abstract:}

In this article we present a detailed study of the diagnosis of the current situation of knowledge management with the use of ICT (Information and communication technology) to teaching-learning process of graduation at the Polytechnic Superior Institute of Kwanza Sul (Angola). The results obtained show a low level of ICT use, low availability of technological resources for knowledge management, limited use and little variety of existing tools and services for the production and management of educational materials, as well as an inadequate information literacy. As data collection tool is used the questionnaire presented by Iriarte (2008), "evaluation of the information management process for the production of content addressed to the teaching-learning process by university teachers" and "assessment of the information management process that is intended to study activity in teaching-learning for university students".

Keywords: knowledge management, formation, higher education institutions

Recibido: 23-06-2014/ Revisado: 21-07-2014/ Aceptado: 20-08-2014/ Publicado: 01-11-2014

https://dx.doi.org/10.25267/Hachetetepe.2014.v2.i9.11 


\section{II: Aprender en la nube}

\section{Introducción}

$\mathrm{L}^{2}$ "Sociedad global de la información" es el término que identifica a la sociedad de finales del siglo XX y comienzos del siglo XXI. Algunos autores como Paradela consideran que más que sociedad o era de la información, es del conocimiento, lo que atribuyen al desarrollo de los soportes que almacenan y distribuyen este. Este autor plantea además que "el desafío de los 80 fue transformar datos en información, el dilema de los 90 fue convertir información en conocimiento y actualmente el desafío podría plantearse en gestionar ese conocimiento" (Paradela, 2001).

«La gestión del conocimiento, en su sentido más amplio, es entendida como el proceso mediante el cual una organización construye nuevo conocimiento y estructura al que ya posee, con el objetivo de que tal conocimiento genere el mayor valor posible para ella y para sus clientes (Páez y Díaz, 2012)»

Las Instituciones de Educación Superior son organizaciones científicas y académicas que generan de forma continua conocimientos, por lo que la asimilación de los vertiginosos cambios que con respecto a las TIC se dan en nuestra era, han permitido que se empleen nuevos métodos para elaborar, adquirir, conservar y transmitir el patrimonio intelectual (García y otros, 2012). Varios estudiosos y teóricos de la Ciencia de la Información (Iriarte, 2008; Torres, 2008), caracterizan este momento como la quinta etapa de su desarrollo, que se enmarca justamente al comienzo de los años sesenta del siglo XX. Esta etapa se caracteriza por la influencia de los avances de las nuevas tecnologías en los métodos de tratamiento y difusión de la información: en primer lugar las computadoras electrónicas (ordenadores) y luego las telecomunica- ciones. Estas y las telecomunicaciones posibilitaron el acceso a la información desde cualquier punto del planeta, cada vez en menos tiempo y con un costo más razonable, que aumenta su eficacia.

Las Tecnologías de la Información y las Comunicaciones (TIC), con sus potencialidades posibilitan la gestión del conocimiento en los diferentes escenarios donde se desarrolla la formación del profesional de la educación (Martínez y Roll, 2012), sin embargo, existe una resistencia enorme a dichos cambios por parte de nuestras instituciones y, muy especialmente, por parte de los profesores que siguen contemplando las TIC como una posibilidad pero no como una parte fundamental del profundo cambio que exigen nuestras aulas universitarias (Roig y Fiorucci, 2010)

Es por ello, que las Universidades precisan crear e implementar tecnologías y sistemas de información que apuesten por la comunicación, que incentiven a los profesores y estudiantes a participar, tomar partido, formar grupo o equipos de trabajo, con el auxilio y dominio de las nuevas tecnologías de información. Estas prácticas deben orientarse a la formación de estrategias para el desarrollo del conocimiento colectivo, el aprendizaje continuo, la comunicación, la colaboración y la búsqueda, así como la generación de información y conocimiento.

En tal sentido, en el presente artículo, se describen los resultados alcanzados en un diagnóstico realizado de la situación actual de la gestión del conocimiento con empleo de las TIC dirigido al proceso de enseñanza aprendizaje de pregrado del Instituto Superior Politécnico de Kwanza Sul en Angola (ISPKS). 


\section{Métodos}

El contexto de estudio correspondió al Instituto Superior Politécnico de Kwansa Sul ubicado en la ciudad de Sumbe, República de Angola, donde se desarrolla el proyecto de investigación "Diseño de un Modelo de Gestión del Conocimiento para el Instituto Superior Politécnico de Kwanza Sul de la República de Angola". Desde la dirección del centro se realizaron las gestiones para poder desarrollar el estudio, el cual se llevó a cabo durante el curso 2013-2014.

\section{Muestra}

La muestra se determinó utilizando la técnica de muestreo no probabilística intencional, ya que permite una cuidadosa y controlada elección de sujetos con posibilidad de brindar mayor información sobre el problema objeto de estudio (Hernández y otros, 2004), o sea permite escoger los integrantes de forma más representativa y con posibilidad de brindar mayor información (Hernández y Coello, 2011). La misma estuvo compuesta por 21 docentes de los que imparten docencia en las carreras del ISPKS, los cuales representan el 33,3\% de una población de 63 docentes efectivos del instituto y 197 de los estudiantes que cursan estudios en las carreras del ISPKS donde imparten clases los docentes encuestados, los cuales representan el 27,6 \% de una población de 715 estudiantes.

\section{Instrumento y procedimiento}

Como herramienta de recogida de datos se emplearon los cuestionarios validados por Iriarte (2008) "Valora- ción del proceso de gestión de información destinada a la producción de contenidos dirigidos al proceso de enseñanza aprendizaje por profesores universitarios" y "Valoración del proceso de gestión de información destinada a la actividad de estudio en el proceso de enseñanza aprendizaje por estudiantes universitarios", ambos fueron adaptados al contexto de la presente investigación. Los cuestionarios que se administraron, en una primera parte solicitaban datos generales de los encuestados, y abarcan las mismas dimensiones que los originales: nivel de utilización de las TIC en el proceso de enseñanza aprendizaje; disponibilidad de recursos tecnológicos para la gestión del conocimiento dirigido al proceso de enseñanza aprendizaje; tipos de materiales producidos para el apoyo al proceso de enseñanza aprendizaje; herramientas y/o servicios para la gestión del conocimiento dirigido al proceso de enseñanza aprendizaje; nivel de precisión de las herramientas y/o servicios para la gestión del conocimiento dirigido al proceso de enseñanza aprendizaje; utilidad de los materiales producidos por otros profesores e instituciones para el apoyo al proceso de enseñanza aprendizaje; elementos que pueden contribuir a optimizar la gestión del conocimiento dirigido al proceso de enseñanza aprendizaje. Cada dimensión contiene preguntas de diferentes tipologías (cerradas $\mathrm{y}$ abiertas en formatos de respuestas únicas y de elección múltiple), cuyos ítems que las integran se detallan a continuación:

- Dimensión nivel de utilización de las TIC en el proceso de enseñanza aprendizaje.

- Grado en que utiliza las TIC en el proceso de enseñanza aprendizaje. (Profesores/Estudiantes). 


\section{II: Aprender en la nube}

- Dimensión disponibilidad de recursos tecnológicos para la gestión del conocimiento dirigido al proceso de enseñanza aprendizaje.

- Grado de disponibilidad de recursos tecnológicos empleados en la gestión de conocimiento y producción de contenidos para el proceso de enseñanza aprendizaje. (Profesores/ Estudiantes).

- Dimensión tipos de materiales producidos para el apoyo al proceso de enseñanza aprendizaje.

- Tipos de materiales elaborados para el apoyo al proceso de enseñanza aprendizaje. (Profesores/Estudiantes).

- Frecuencia de participación en la elaboración conjunta de materiales educativos con otros profesores e instituciones dirigidos al proceso de enseñanza aprendizaje. (Profesores).

- Frecuencia con que personaliza y/o adapta el material que está elaborando, a las características de un estudiante o grupo de estudiantes de un entorno determinado. (Profesores).

- Dimensión herramientas y/o servicios para la gestión del conocimiento dirigido al proceso de enseñanza aprendizaje.

- Grado de empleo de herramientas de autor para la elaboración de contenidos dirigidos al proceso de enseñanza aprendizaje. (Profesores).

- Herramientas y/o servicios utilizados para publicar y gestionar los materiales dirigidos al proceso de enseñanza aprendizaje. (Profesores/ Estudiantes).

- Forma de clasificación utilizada para publicar los materiales en algún sistema. (Profesores).

- Dimensión nivel de precisión de las herramien- tas y/o servicios para la gestión del conocimiento dirigido al proceso de enseñanza aprendizaje.

- Herramientas y/o servicios mediante los que logra localizar materiales dirigidos al proceso de enseñanza aprendizaje. (Profesores/Estudiantes).

- Grado de precisión en los resultados de búsqueda cuando emplea herramientas y/o servicios para la gestión del conocimiento dirigido al proceso de enseñanza aprendizaje. (Profesores/ Estudiantes).

- Tipos de información gestionada utilizando los servicios de Internet para el apoyo al proceso de enseñanza aprendizaje. (Profesores/ Estudiantes).

- Utilidad de los materiales producidos por otros profesores e instituciones para el apoyo al proceso de enseñanza aprendizaje.

- Nivel de utilidad de la reutilización de materiales educativos elaborados por otros profesores e instituciones dirigidos al proceso de enseñanza aprendizaje. (Profesores/Estudiantes).

- Frecuencia con que emplea solo una parte o fragmento de un material educativo realizado por otro profesor en el proceso de producción de contenidos para el apoyo al proceso de enseñanza aprendizaje. (Profesores).

- Limitaciones para lograr la selección de un fragmento o parte de un material educativo elaborado por otro profesor o institución para incluir en sus clases y cursos dirigidos al proceso de enseñanza aprendizaje. (Profesores).

- Elementos que pueden contribuir a optimizar la gestión del conocimiento dirigido al proceso de enseñanza aprendizaje. (Profesores/Estudiantes). 


\section{Análisis de los datos}

Para el análisis de los datos se empleó la estadística descriptiva mediante el análisis porcentual, y se representaron los resultados en forma de gráficos para un mejor análisis e interpretación. El análisis de los datos se realizó a través del programa estadístico SPSS 14.0 .

\section{Resultados}

Después de los análisis estadísticos realizados, se obtienen los resultados que a continuación presentamos, en relación con las valoraciones de los docentes y estudiantes respecto a las siete dimensiones en estudio.

\section{Resultados obtenidos del cuestionario aplicado a los docentes del ISPKS}

La caracterización académica y científica de los docentes encuestados dio como resultado que el 57,1\% ostentan categorías docentes de Profesor Auxiliar y Titular, el $85,7 \%$ posee el grado científico de doctor en ciencias de determinada especialidad $(\mathrm{PhD})$ o el grado académico de maestros en ciencias (Magister), el 76,2\% lleva más de 10 años de experiencia como docente; razón por la cual se puede considerar un claustro experimentado con un adecuado nivel académico y científico.

En términos generales, los resultados alcanzados para la dimensión "nivel de utilización de las TIC en el proceso de enseñanza aprendizaje", demostraron un bajo nivel de utilización, pues solo el 57,1\% de los docentes refirió un nivel muy alto o alto de utilización de las TIC en la docencia. Los mismos están correlacionados con los que se obtienen para la dimensión "disponibilidad de recursos tecnológicos para la gestión del conocimiento dirigido al proceso de enseñanza aprendizaje" (Figura 1), donde el 85,7\% de los docentes enunciaron, una baja o nula disponibilidad de equipos de digitalización, el 66,7\% destacó una baja disponibilidad y uso de dispositivos móviles y un $57,1 \%$ de computadoras para la gestión del conocimiento y la producción de contenidos para el proceso de enseñanza aprendizaje. Además, se debe destacar que el 57,1\% planteó que el acceso a Internet es bajo o nulo.

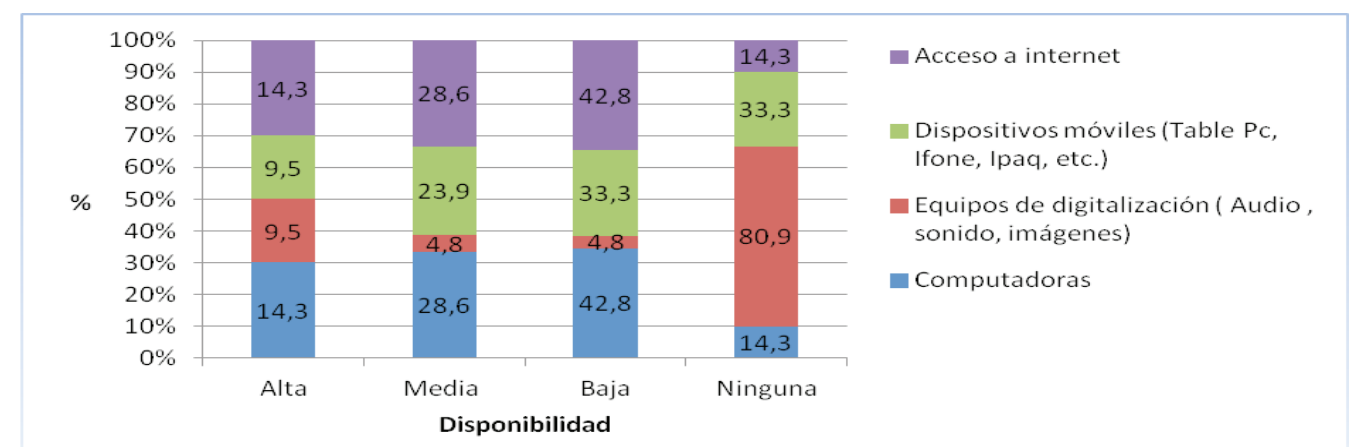

Figura 1. Disponibilidad de recursos tecnológicos empleados por los docentes en la gestión de conocimiento y producción de contenidos para el proceso de enseñanza aprendizaje. Fuente: Elaboración propia 


\section{IIA Aprender en la nube}

$\mathrm{Al}$ analizar las respuestas dadas por los docentes en relación con la dimensión "tipos de materiales producidos para el apoyo al proceso de enseñanza aprendizaje", el $100 \%$ enunciaron, en primera opción, el uso de presentaciones (Power Point) como material elaborado para la docencia en las diferentes asignaturas y el $66,7 \%$ el uso del texto. En el caso de otros materiales, se ubicaron en el orden de prioridad la multimedia $(28,6 \%)$ y los vídeos e imágenes $(23,1 \%)$ respectivamente (Figura 2). Además, se determinó una baja frecuencia de participación de los docentes del ISPKS en la elaboración conjunta de materiales educativos con otros profesores o instituciones, ya que el $57,2 \%$ pocas veces o nunca lo han hecho, así como una baja frecuencia de personalización y/o adaptación de los materiales elaborados a las características de un estudiante o grupo de estudiantes de un entorno determinado, ya que el $61,9 \%$ pocas veces o nunca lo han hecho.

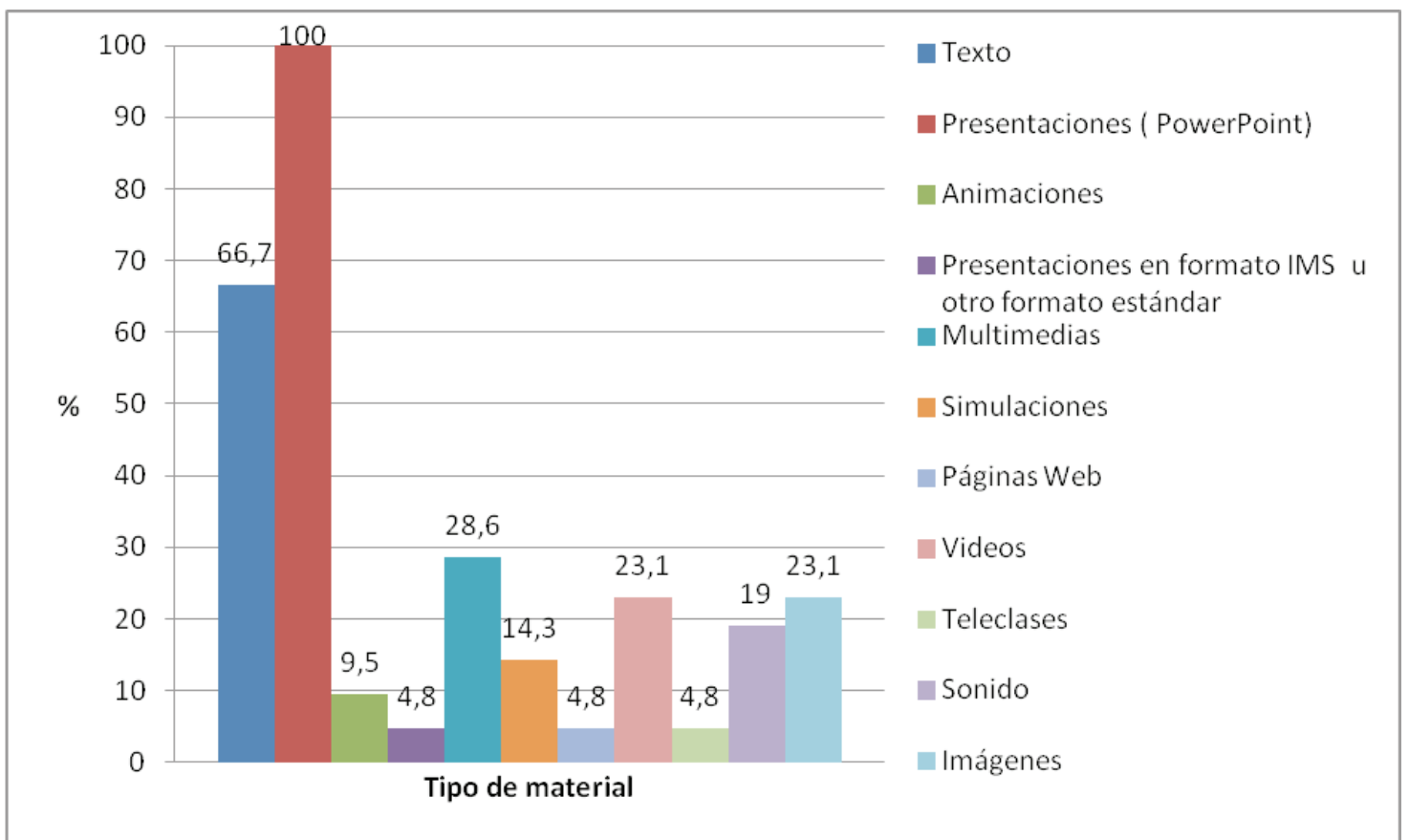

Figura 2. Tipos de materiales elaborados utilizando las TIC por los docentes para el apoyo al proceso de enseñanza aprendizaje Fuente: Elaboración propia 
Los resultados correspondientes a la dimensión "herramientas y/o servicios para la gestión del conocimiento dirigido al proceso de enseñanza aprendizaje", manifestaron un grado de empleo de herramientas de autor para la elaboración de contenidos dirigidos al proceso de enseñanza aprendizaje, predominando el uso del editor de presentaciones de Power Point (52,3\%) y el de editores de texto (47,7\%) (Figura 3). En relación con las herramientas y/o servicios utilizados para publicar y gestionar los materiales dirigidos al proceso de enseñanza aprendizaje, los docentes encuestados señalaron como las más utilizadas el envío por correo electrónico a otros profesores $(66,7 \%)$, las carpetas y archivos compartidos $(52,4 \%)$ y los CD, DVD u otro medio similar (42,8\%), lo cual demuestra un uso limitado por parte de los profesores de la variedad de herramientas y servicios existentes para la producción y gestión de materiales educativos (Figura 4). Por último, en la dimensión analizada se indagó respecto a las formas empleadas para clasificar los materiales educativos para ser publicados en algún sistema, o sea si utilizaban algún sistema de catalogación, lo cual arrojó como resultado que el 85,7 de los docentes encuestados no catalogan sus materiales educativos que producen, lo cual demuestra la no existencia de un sistema de catalogación de los recursos destinados al proceso de enseñanza aprendizaje en el ISPKS.

En los resultados relacionados a la dimensión "nivel de precisión de las herramientas y/o servicios para la gestión del conocimiento dirigido al proceso de enseñanza aprendizaje", se identificaron como herramientas y/o servicios más utilizados para localizar materiales dirigidos al proceso de enseñanza aprendizaje, los buscadores de Internet $(71,5 \%)$ y el intercambio por correo electrónico (52,4\%) (Figura 5), así como la de mayor grado de precisión los buscadores de Internet para (61,9\%) (Figura 6). Además, los docentes destacaron como tipos de información más gestionada utilizando los servicios de Internet para el apoyo al proceso de enseñanza aprendizaje: la información científica (80,9\%); las revistas científicas $(71,5 \%)$; y los contenidos educativos, Sitios Web propios del área del conocimiento y noticias internacionales $(47,6 \%)$ (Figura 7). 


\section{II: Aprender en la nube}

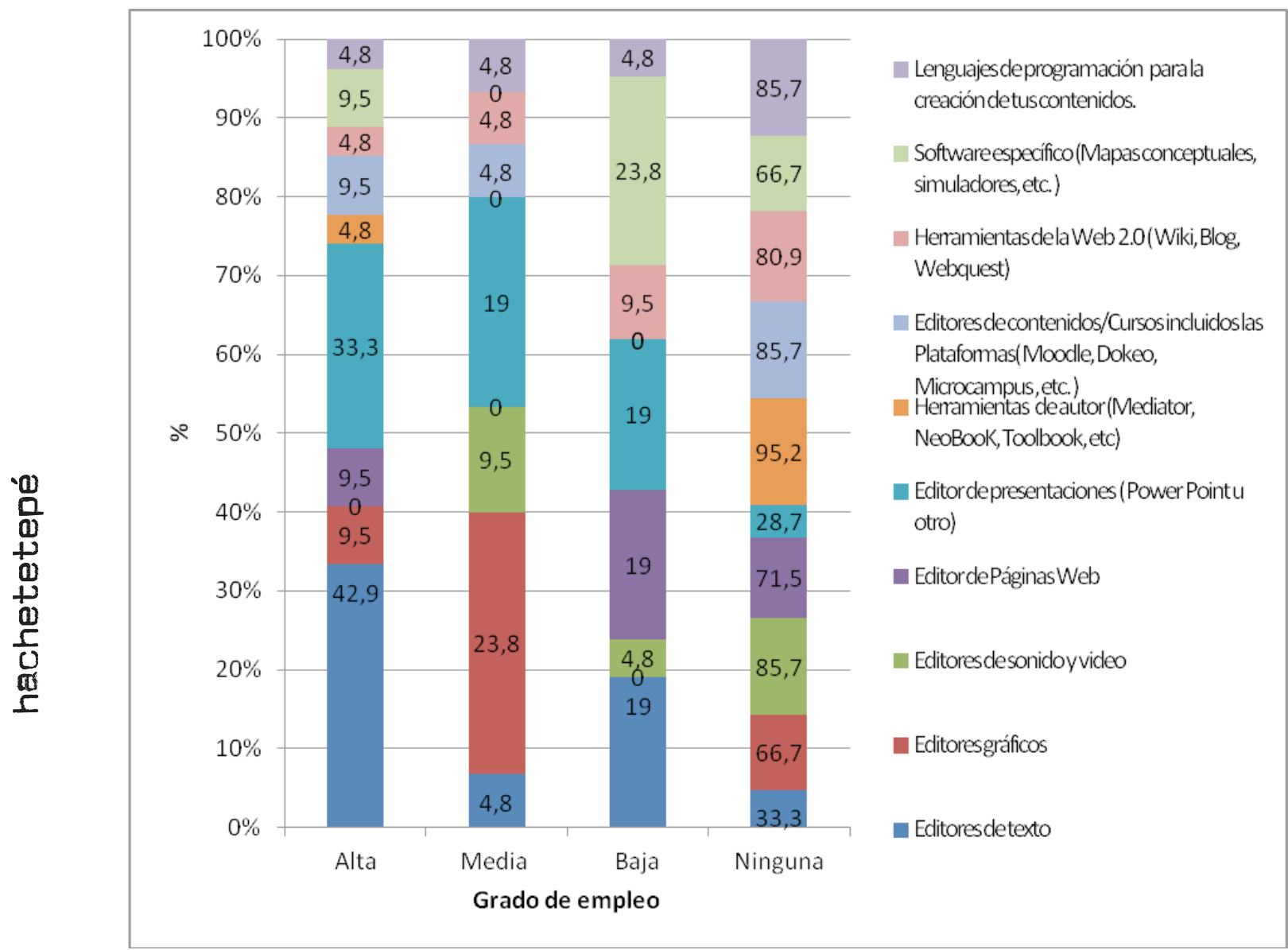

Figura 3. Grado de empleo de herramientas de autor por los docentes para la elaboración de contenidos dirigidos al proceso de enseñanza aprendizaje. Fuente: Elaboración propia 
Experiencia de un diagnóstico sobre la situación actual del Instituto Superior Politécnico de Kwanza Sul (Angola) en la gestión del conocimiento con empleo
de las TIC dirigido al proceso de enseñanza aprendizaje

\section{Divulgatio}

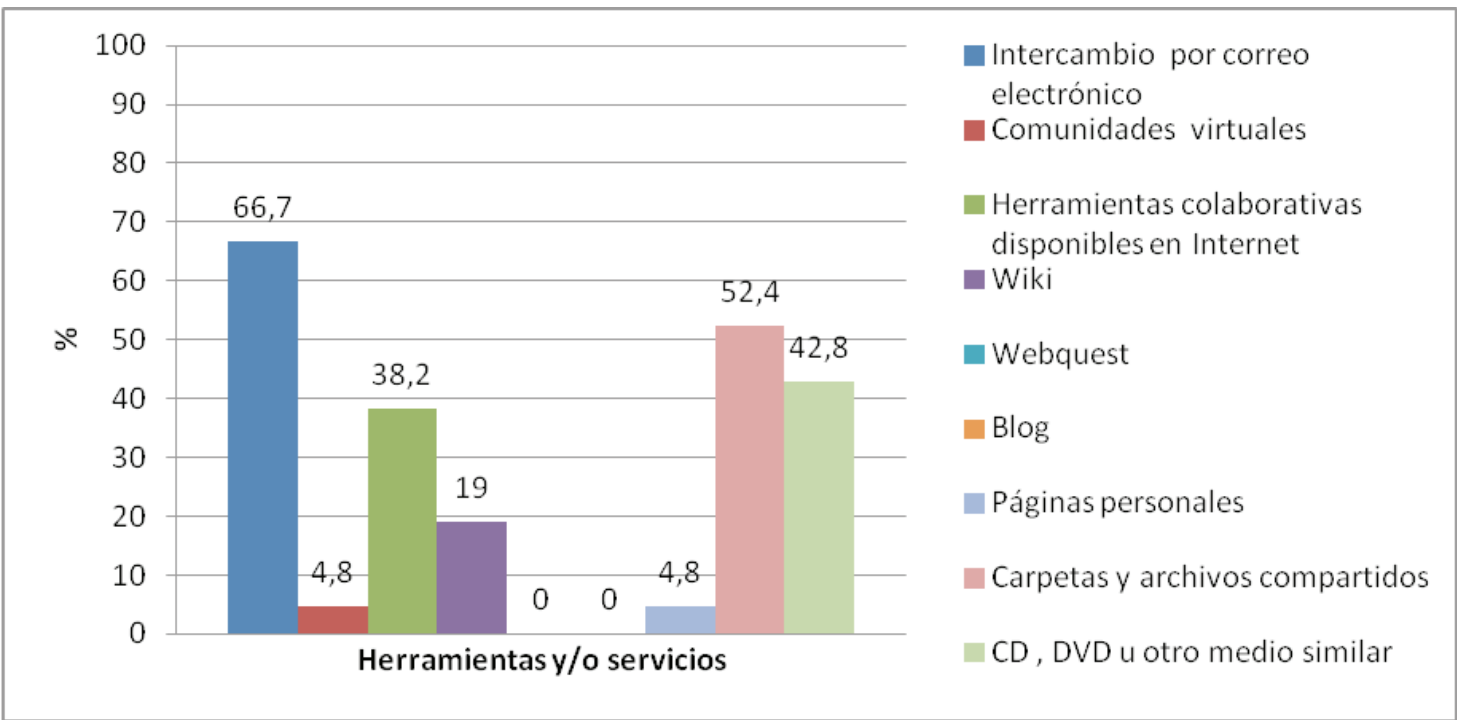

Figura 4. Herramientas y/o servicios utilizados por los docentes para publicar y gestionar los materiales dirigidos al proceso de enseñanza aprendizaje.. Fuente: Elaboración propia

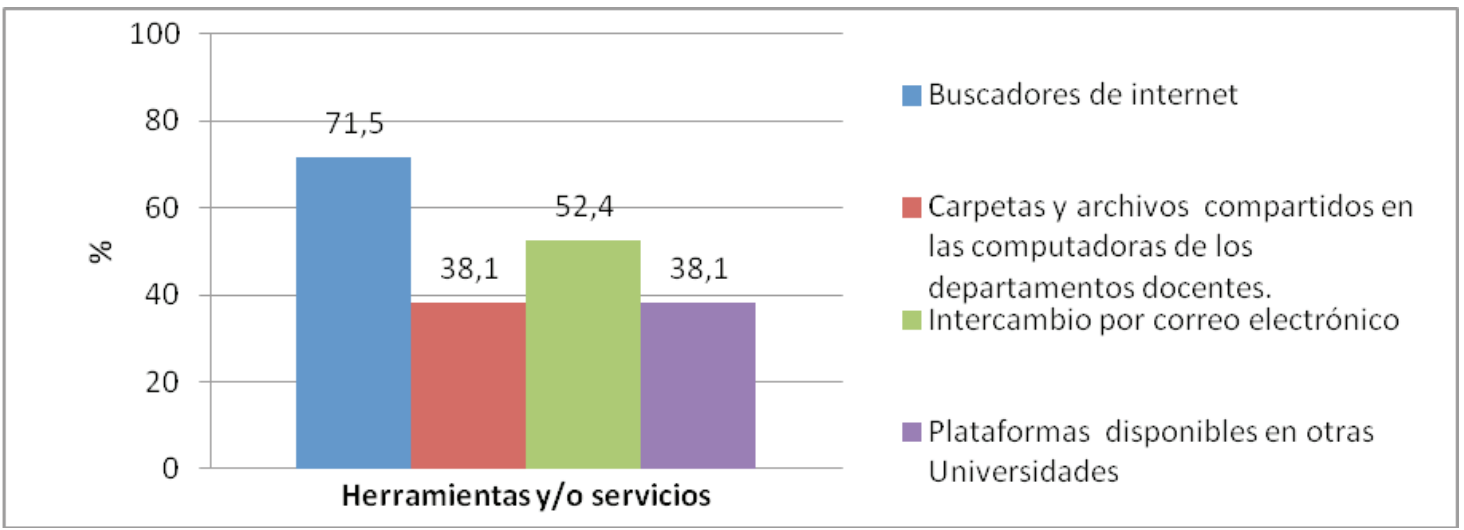

Figura 5. Herramientas y/o servicios mediante los que los docentes logran localizar materiales dirigidos al proceso de enseñanza aprendizaje. Fuente: Elaboración propia 


\section{II: Aprender en la nube}

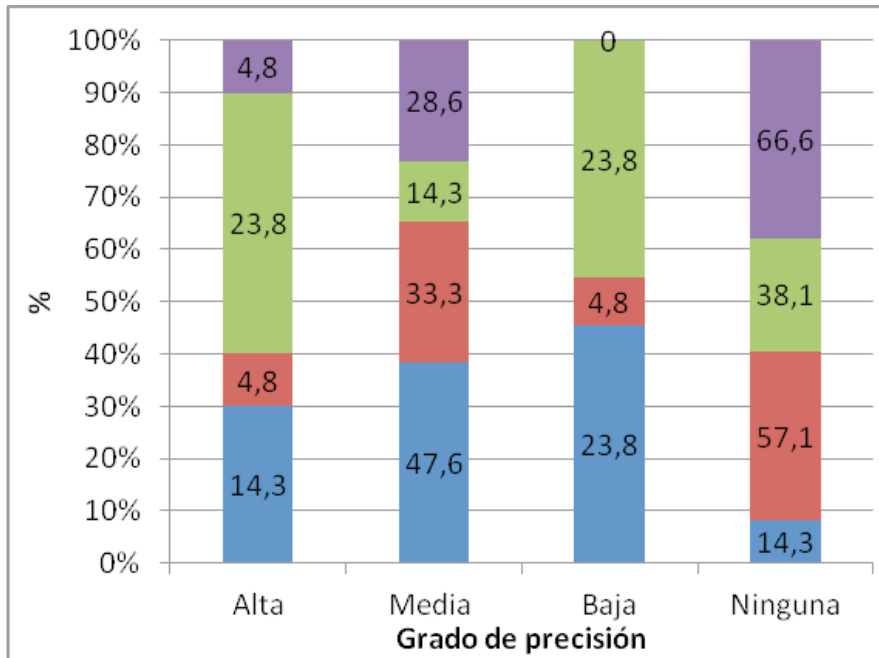

Plataformas disponibles en otras Universidades

- Intercambio por correo electrónico

- Carpetas y archivos compartidos en las computadoras de los departamentos docentes.

Buscadores de internet

Figura 6. Grado de precisión en los resultados de búsqueda de los docentes cuando emplea herramientas y/o servicios para la gestión del conocimiento dirigido al proceso de enseñanza aprendizaje. Fuente: Elaboración propia

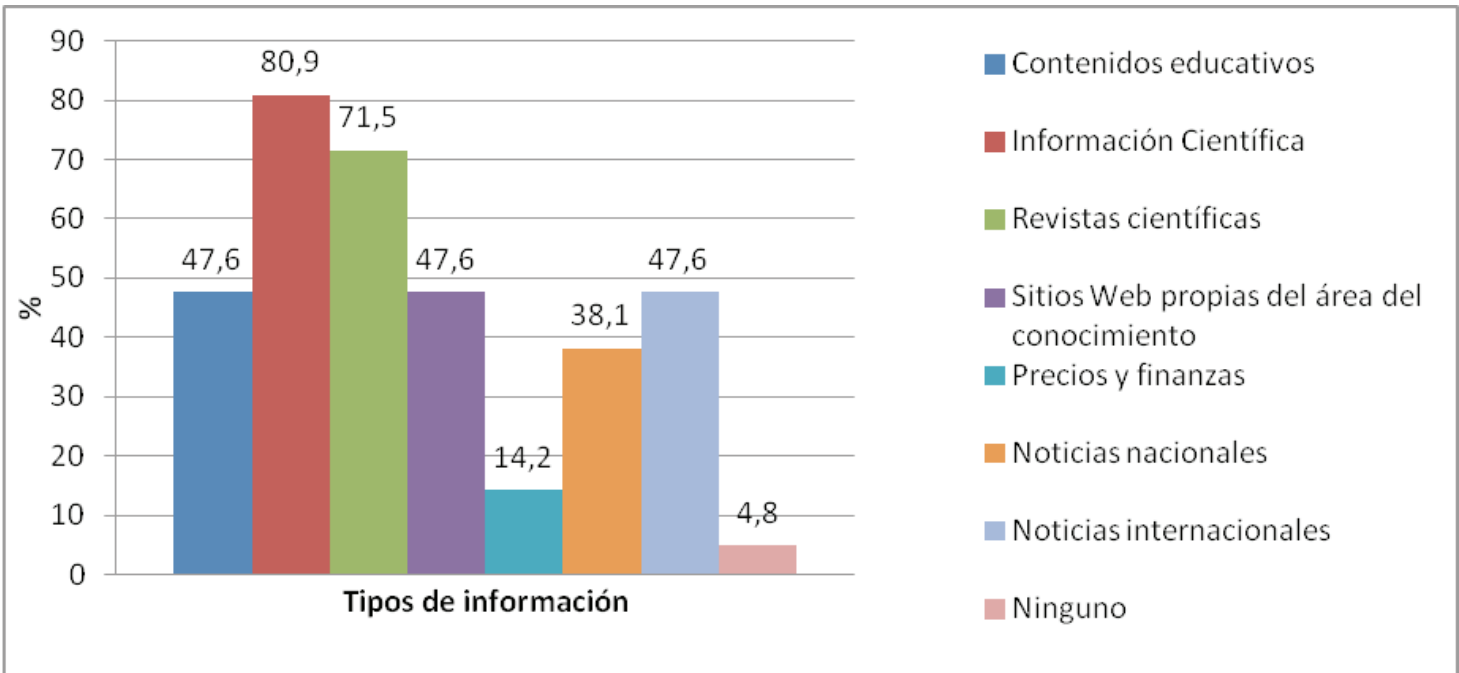

Figura 7. Tipos de información más gestionada por los docentes utilizando los servicios de Internet para el apoyo al proceso de enseñanza aprendizaje. Fuente: Elaboración propia 
Los resultados relacionados con la dimensión "utilidad de los materiales producidos por otros profesores e instituciones para el apoyo al proceso de enseñanza aprendizaje", refieren que el 90,5\% de los docentes encuestados los considera de utilidad, pero solo el $42,8 \%$ plantean haber reutilizado una parte o fragmento de un material educativo realizado por otro profesor en el proceso de producción de contenidos para el apoyo al proceso de enseñanza aprendizaje. Entre las limitaciones fundamentales para lograr la selección de un fragmento o parte de un material educativo elaborado por otro profesor o institución para incluir en sus clases se identifican: no se puede editar el recurso fácilmente $(57,1 \%)$; muy tedioso el proceso de edición cuando lo materiales son muy extensos (19\%); y el recurso no ofrece una estructura que permita buscar y seleccionar fácilmente (19\%).

Finalmente, se analizan los resultados de la dimensión "elementos que pueden contribuir a optimizar la gestión del conocimiento dirigido al proceso de enseñanza aprendizaje" mediante el empleo de una pregunta abierta, para la cual los comentarios y sugerencias de los profesores, identificaron como los elementos que pueden optimizar la producción de materiales educativos para el proceso de enseñanza los siguientes: disponibilidad de computadoras $(66,7 \%)$; acceso a Internet $(61,9 \%)$; herramientas y revistas científicas disponibles (19\%); existencia de un Sitio Web del ISPKS $(9,5)$; tiempo de trabajo en la biblioteca e intercambio con otras Universidades $(4,8 \%)$.

\section{Resultados obtenidos del cuestionario aplicado a los estudiantes del ISPKS}

Los resultados de la encuesta realizada a los profeso- res fueron, a su vez, contrastados con las respuestas ofrecidas por los estudiantes encuestados, posibilitando llegar a la determinación de un grupo de insuficiencias en relación con el comportamiento de la problemática analizada.

La caracterización de los estudiantes, arrojó como resultado que el 58,9\% son del sexo masculino y $41,1 \%$ del sexo femenino, con una edad promedio de 25,6 años, y se logró encuestar los estudiantes de todas las carreras que recibían docencia con los profesores encuestados para la presente investigación; razón por la cual se pueden establecer análisis comparativos de interés entre los resultados de ambos cuestionarios con vistas a establecer regularidades.

En términos generales, los resultados alcanzados para la dimensión "nivel de utilización de las TIC en el proceso de enseñanza aprendizaje", demostraron un bajo nivel de utilización, pues solo el 33\% de los estudiantes refirió un nivel muy alto o alto de utilización de las TIC en la docencia. Los mismo están correlacionados con los que se obtienen para la dimensión "disponibilidad de recursos tecnológicos para la gestión del conocimiento dirigido al proceso de enseñanza aprendizaje" (Figura 8), donde el 92,4\% de los estudiantes enunciaron, una baja o nula disponibilidad de equipos de digitalización, el 90,9\% destacó una baja disponibilidad y uso de dispositivos móviles y un 69\% de computadoras para la gestión del conocimiento para el proceso de enseñanza aprendizaje. Además, se debe destacar que el 88,3\% planteó que el acceso a Internet es bajo o nulo. Estos resultados reafirman el bajo nivel de utilización de las TIC en el proceso de enseñanza aprendizaje identificado por los docentes.

Al analizar las respuestas dadas por los estudiantes en relación con la dimensión "tipos de materiales produ- 


\section{II: Aprender en la nube}

cidos para el apoyo al proceso de enseñanza aprendizaje", el 62,9\% enunciaron, en primera opción, el uso del texto como material elaborado por los docentes para el proceso de enseñanza aprendizaje, y el 33,5\% el uso de las presentaciones (Power Point). En el caso de otros materiales, se ubicaron en el orden de prioridad las imágenes $(23,1 \%)$ (Figura 9).

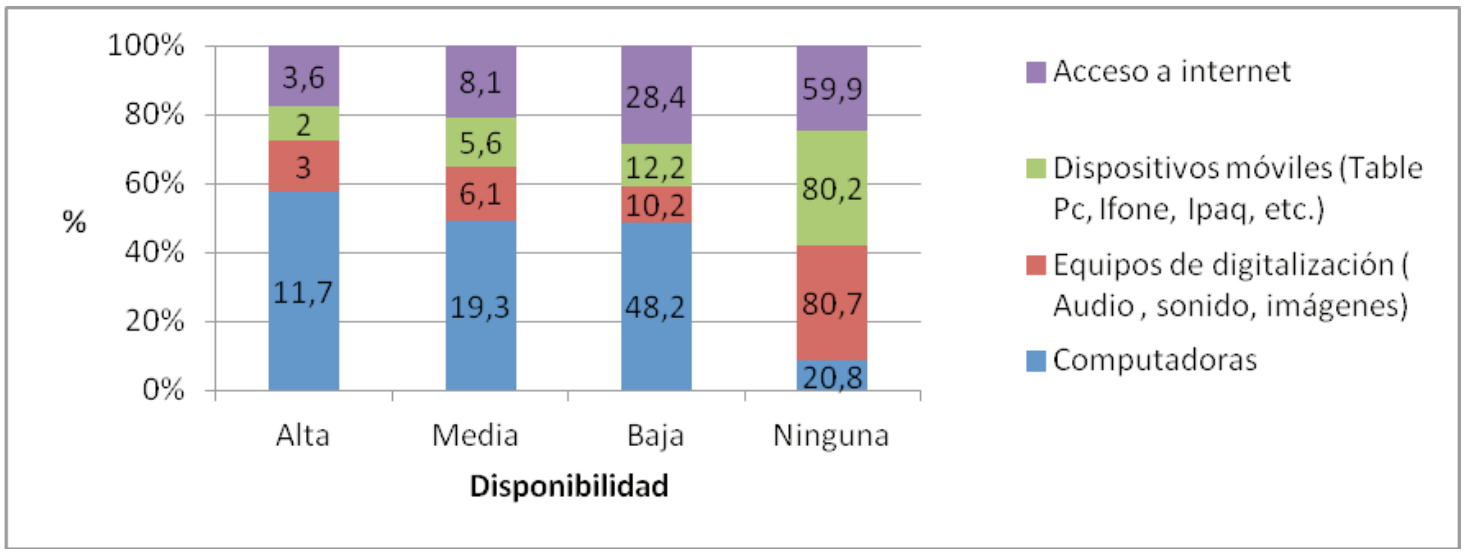

Figura 8. Disponibilidad de recursos tecnológicos empleados por los estudiantes en la gestión de conocimiento para el proceso de enseñanza aprendizaje

Fuente: Elaboración propia 


\section{Divulgatio}

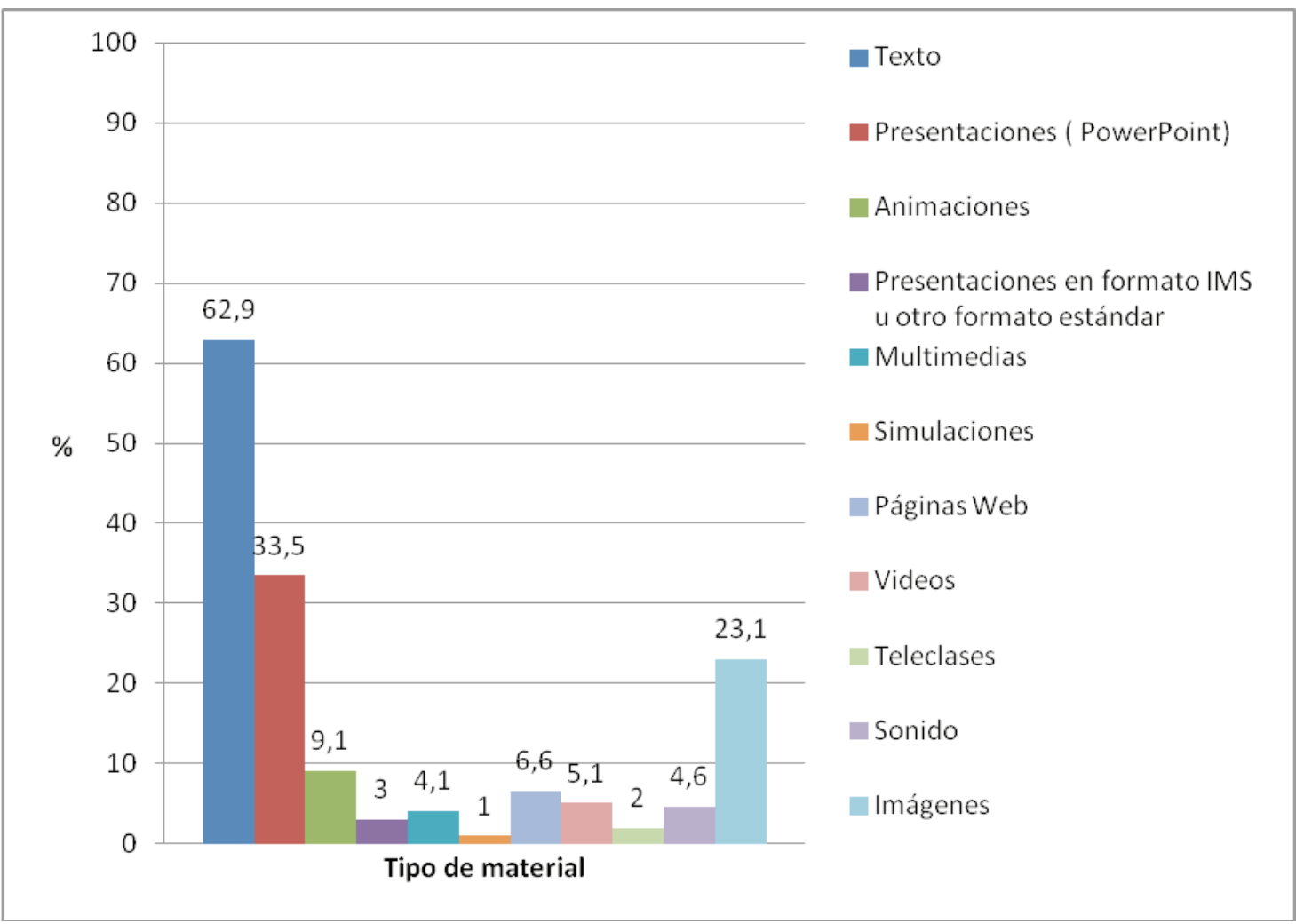

Figura 9. Tipos de materiales elaborados utilizando las TIC empleados por los estudiantes en el proceso de enseñanza aprendizaje. Fuente: Elaboración propia

Los resultados correspondientes a la dimensión "herramientas y/o servicios para la gestión del conocimiento dirigido al proceso de enseñanza aprendizaje", en relación con las herramientas y/o servicios utilizados para gestionar los materiales dirigidos al proceso de enseñanza aprendizaje, los estudiantes encuestados señalaron como las más utilizadas las herramientas colaborativas disponibles en Internet $(31,5 \%)$, los
CD, DVD u otro medio similar $(23,4 \%)$ y las carpetas y archivos compartidos (52,4\%), lo cual demuestra un uso limitado por parte de los estudiantes de la variedad de herramientas y servicios existentes para la gestión de materiales educativos (Figura 10), resultados estos que reafirman los alcanzados en el cuestionario a docentes. 


\section{IIA Aprender en la nube}

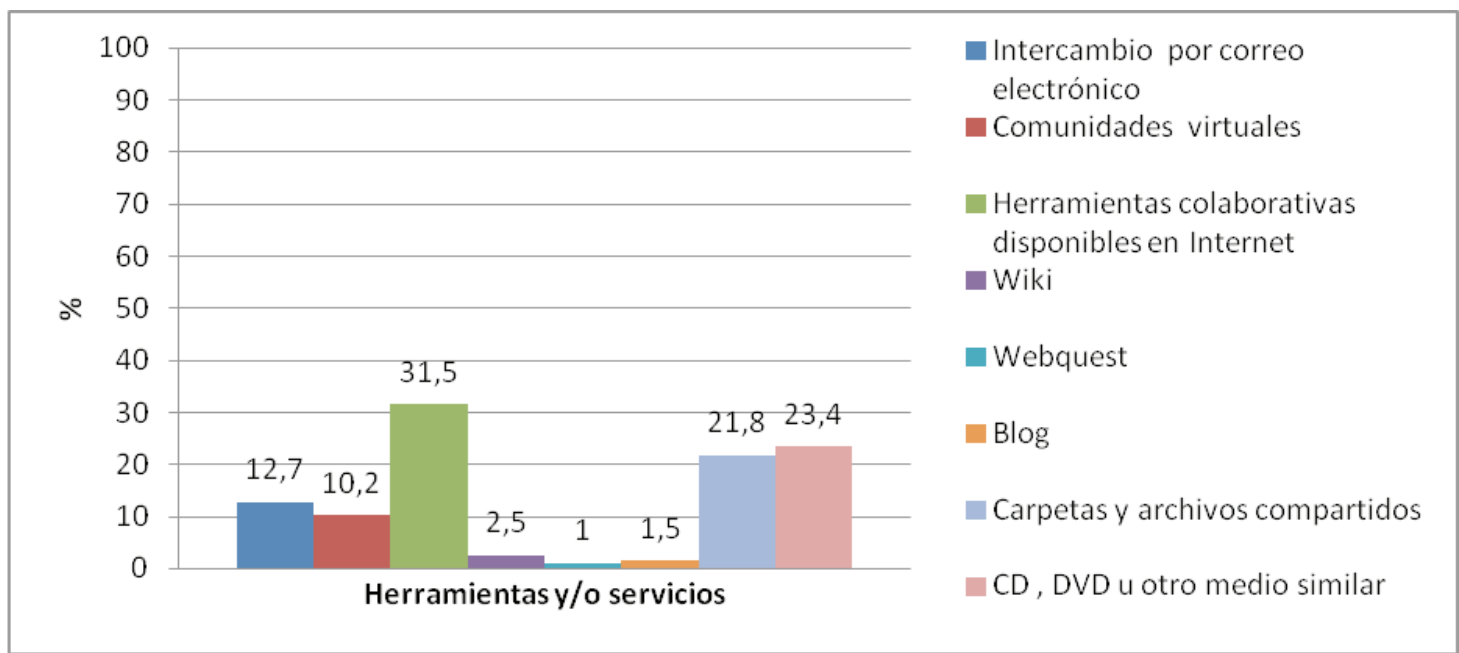

Figura 10. Herramientas y/o servicios utilizados por los estudiantes para gestionar los materiales dirigidos al proceso de enseñanza aprendizaje. Fuente: Elaboración propia

En los resultados relacionados con la dimensión "nivel de precisión de las herramientas y/o servicios para la gestión del conocimiento dirigido al proceso de enseñanza aprendizaje", se identificaron como herramientas y/o servicios más utilizados para localizar materiales dirigidos al proceso de enseñanza aprendizaje, los buscadores de Internet $(48,7 \%)$ y las carpetas y archivos compartidos en las computadoras de los departamentos docentes (21,8\%) (Figura 11), así como la de mayor grado de precisión los buscadores de Internet (27,8\%) (Figura 12), lo cual demuestra el bajo nivel de uso y precisión que poseen los estudiantes para utilizar las TIC en su proceso de enseñanza aprendizaje. Además, los estudiantes destacaron como tipos de información más gestionada utilizan- do los servicios de Internet para el apoyo al proceso de enseñanza aprendizaje: los contenidos educativos $(57,4 \%)$; la información científica $(41,6 \%)$; y las noticias internacionales $(28,4 \%)$ y nacionales $(26,9 \%)$ (Figura 13).

Los resultados relacionados con la dimensión "utilidad de los materiales producidos por otros profesores e instituciones para el apoyo al proceso de enseñanza aprendizaje", refieren que el $77,1 \%$ de los estudiantes encuestados los considera de utilidad.

Finalmente, se analizan los resultados de la dimensión "elementos que pueden contribuir a optimizar la gestión del conocimiento dirigido al proceso de enseñanza aprendizaje" mediante el empleo de una pregunta abierta, para la cual los comentarios y sugerencias de 
Experiencia de un diagnóstico sobre la situación actual del Instituto Superior Politécnico de Kwanza Sul (Angola) en la gestión del conocimiento con empleo de las TIC dirigido al proceso de enseñanza aprendizaje

\section{Divulgatio}

los estudiantes, identificaron como los elementos que pueden optimizar la gestión de materiales educativos para el proceso de enseñanza los siguientes: disponibilidad de computadoras (67,5\%); acceso a Internet de banda ancha (60,4\%); construcción de una Intranet del ISPKS (38,1\%); incrementar el número de laboratorios de informática (26,4\%); y disponibilidad de una Biblioteca Digital y un Repositorio de Objetos de Aprendizaje en la Intranet del ISPKS (17,8\%).

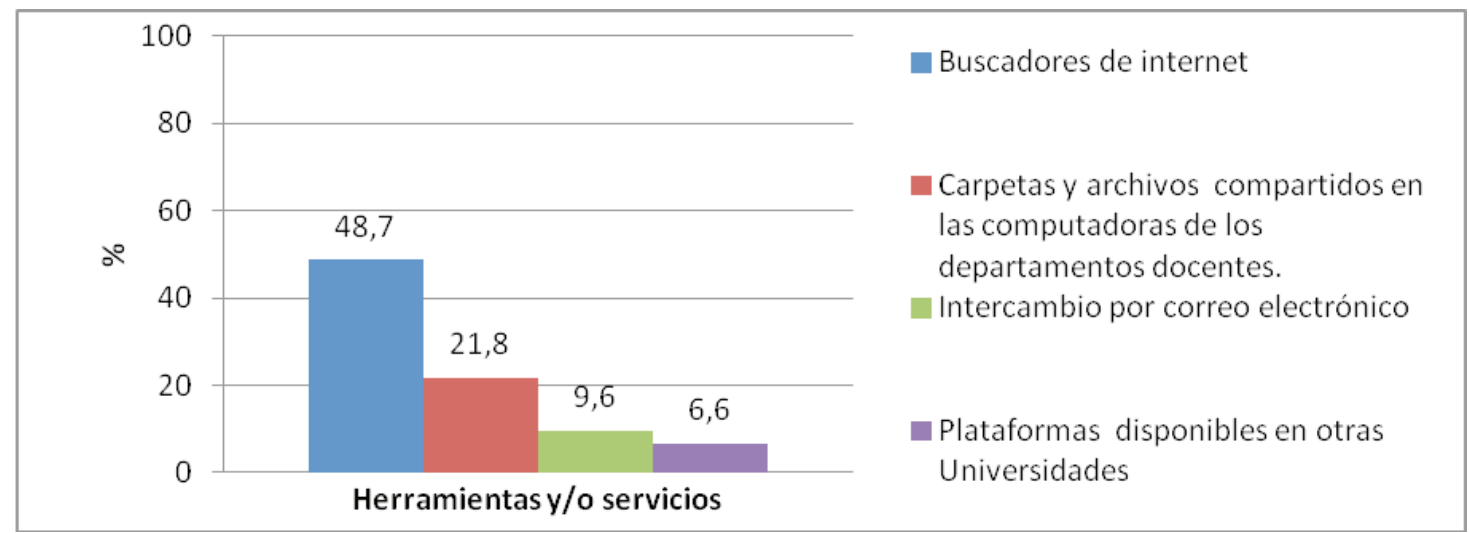

Figura 11. Herramientas y/o servicios mediante los que los estudintes logran localizar materiales dirigidos al proceso de enseñanza aprendizaje

Fuente: Elaboración propia 


\section{II: Aprender en la nube}

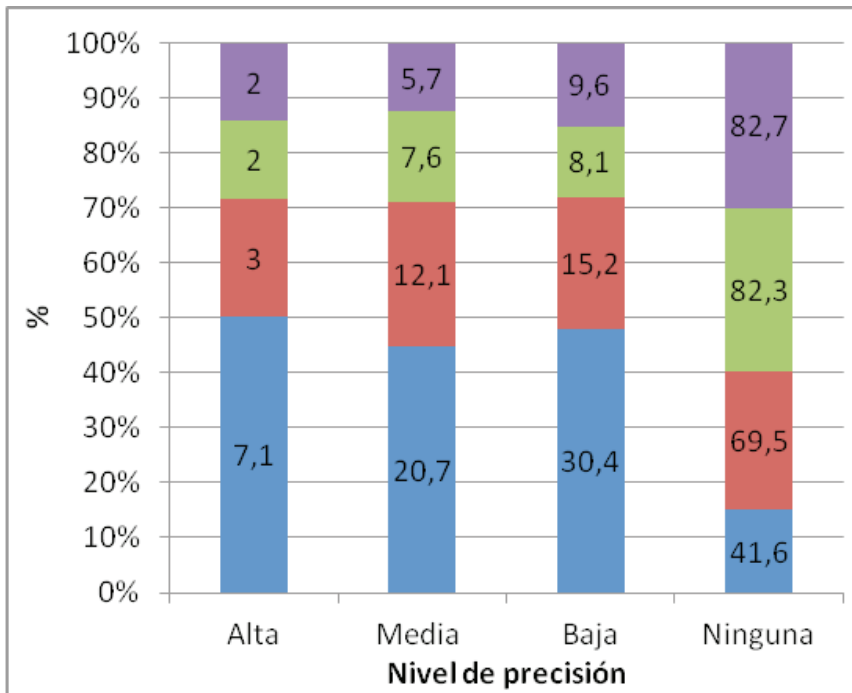

Plataformas disponibles en otras

Universidades

- Intercambio por correo electrónico

- Carpetas y archivos compartidos en las computadoras de los departamentos docentes.

Buscadores de internet

Figura 12. Grado de precisión en los resultados de búsqueda de los estudiantes cuando emplea herramientas y/o servicios para la gestión del conocimiento dirigido al proceso de enseñanza aprendizaje. Fuente: Elaboración propia

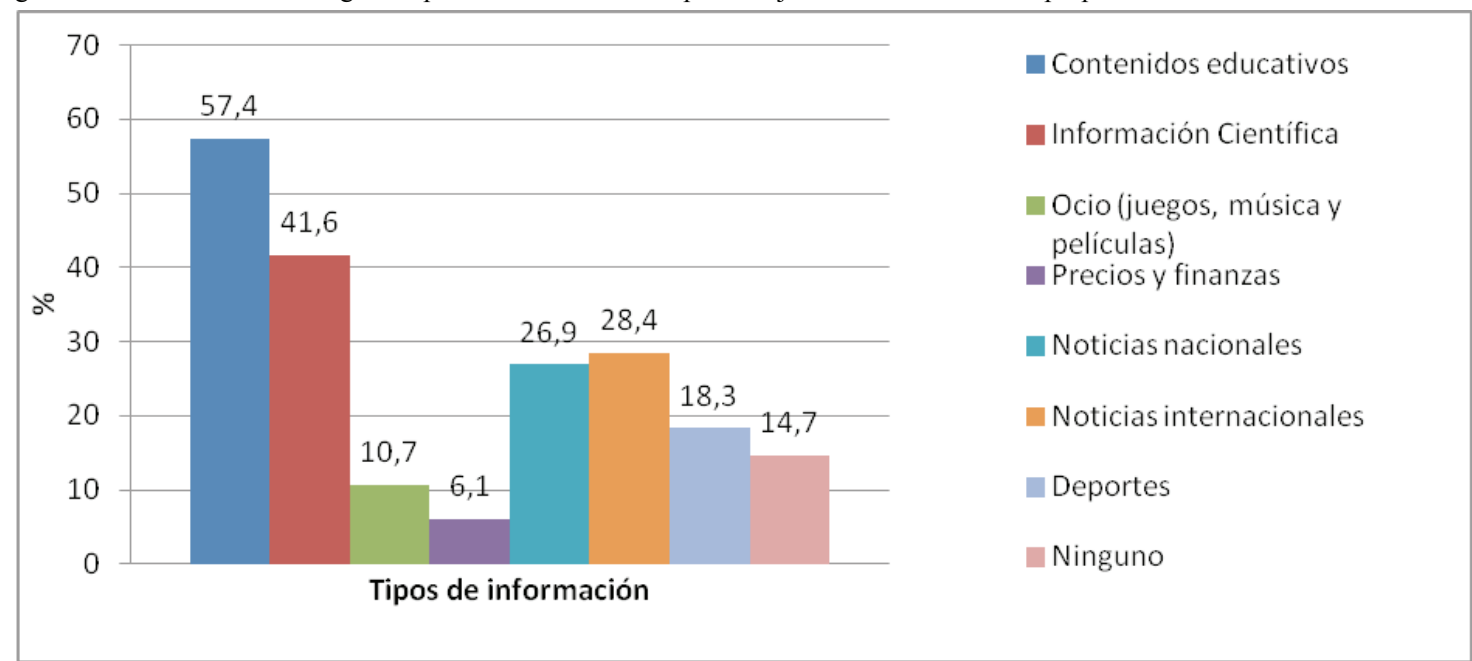

Figura 13. Tipos de información más gestionada por los estudiantes utilizando los servicios de Internet para el apoyo al proceso de enseñanza aprendizaje. Fuente: Elaboración propia 


\section{Discusión y conclusión}

A partir de los resultados expuestos es posible concluir que existe un bajo nivel de utilización de las TIC en el ISPKS, así como una baja disponibilidad de recursos tecnológicos para la gestión del conocimiento dirigido al proceso de enseñanza aprendizaje, puesto al relieve en los criterios de los docentes y estudiantes encuestados, por lo que dicha institución, teniendo en cuenta la clasificación propuesta en el informe realizado por la CRUE en 2006 citada por Prendes (2011), donde se agrupan las Universidades en función de la incorporación que cada una de ellas hace de las TIC, se ubica en el grupo de las Universidades autosuficientes, caracterizadas por una integración discreta de las TIC, gran escepticismo por parte de los docentes y ausencia de liderazgo impulsor.

En correspondencia directa con el nivel de incorporación de las TIC que ubica al instituto en el grupo de Universidades antes mencionado, se corroboró que entre los factores que influyen se destaca la falta de una infraestructura clara y eficiente, acompañada de un déficit de formación del docente, criterios que coinciden con los expresado por Espuny y otros (2012), así como la falta de alfabetización digital de los estudiantes, los cuales "son menos competentes tecnológicamente hablando de lo que nos podemos imaginar y hacen más utilización mecánica e intuitiva de las tecnologías que consiente y dirigida" (Cabero y otros, 2011).

Los docentes y estudiantes demuestran un uso limitado y poco preciso de la variedad de herramientas y servicios existentes para la producción y gestión de materiales educativos, desaprovechando las posibilidades tecnológicas que brinda la existencia de la Web
2.0, lo cual evidencia que la formación en TIC de los docentes para el uso y dominio en apoyo a las actividades académicas, es muy deficiente y en algunos casos nula coincidiendo con los resultados alcanzados por Rosario y Vásquez (2012), la cual repercute de manera directa en los estudiantes.

En cuanto al manejo de estándares, gestores de información y herramientas para la colaboración en redes sociales, los docentes y estudiantes encuestados evidencian una inadecuada alfabetización informacional para llevar a cabo la elaboración y gestión de los recursos destinados al proceso de enseñanza aprendizaje en el ISPKS, de forma que faciliten su gestión, disponibilidad y reusabilidad tal como expresan Iriarte (2008) y Nápoles (2011) en sus investigaciones.

Los resultados hasta aquí analizados, reafirman los alcanzados por Da Silva y otros (2013), en relación con el nivel de desarrollo de los sistemas de gestión del conocimiento dirigido al proceso de formación de profesionales con base a criterios de naturaleza técnico-pedagógica, donde el Instituto Superior Politécnico de Kwanza Sul clasifica en el Nivel I.

Por todo lo anteriormente expresado, se considera necesario, tal como expresan Roig y Flores (2014), la ejecución de grandes inversiones en equipamiento tecnológico e infraestructuras y la formación del profesorado como factor decisivo en la integración de las TIC en los centros educativos, así como la formación de los estudiantes en el "uso y consumo de las nuevas herramientas que hoy se han denominado 2.0" (Cabero y otros, 2011) con vistas a perfeccionar la gestión del conocimiento dirigido al proceso de enseñanza aprendizaje para el ISPKS. 


\section{II: Aprender en la nube}

\section{Referencias}

Cabero, J.; Llorente, M. C. y Marín, V. (2011). "Las prácticas con TIC: el acercamiento a la sociedad del conocimiento ¿Están los alumnos capacitados?, en Roig R. y Laneve C. (Coords.). La práctica educativa en la Sociedad de la Información. Innovación a través de la investigación. Editorial Marfil; 80.

Da Silva, A.; López, A. y Torres, A. (2013). “Tendencias actuales en la gestión del conocimiento en las Instituciones de Educación Superior públicas de la República de Angola". Revista IPLAC, 5. (http://www. revista.iplac.rimed.cu/index.php?option $=$ com_conten t\&view $=$ category \&id=34\&IteIte=338) (Recuperado el 21 de abril de2014).

Espuny, C.; Gisbert, M. y otros (2012). "El coordinador TIC en los Centros educativos: funciones para la dinamiezación e incorporación didáctica de las TIC en las actividades de aprendizaje". Pixel-Bit, 41; 7-18.

García, M.; Salazar, A. I. y Batista, M. (2012). "Repositorios digitales. Incremento de la visibilidad y el impacto de la ciencia cubana". Revista IPLAC, 4. (http:// www.revista.iplac.rimed.cu/index.php?option $=$ com content\&view=category\&id=34\&Itemid=270). $\quad(\mathrm{Re}-$ cuperado el 21 de abril de 2014).

Hernández, R.; Fernández, C. y Baptista, P. (2004) Metodología de la Investigación. La Habana: Editorial Félix Valera.

Hernández, R. A. y Coello, S. (2011) El proceso de la investigación cientifica. Ciudad de la Habana: Editorial Universitaria.

Iriarte, L. E. (2008). Modelo para la gestión de información en la producción de contenidos didácticos en la Nueva Universidad Cubana. Tesis en opción al Grado Científico de Doctor en Ciencias de la Educación.
Universidad Agraria de La Habana.

Martínez, R. y Roll, M. (2012). "Estrategia curricular infotecnológica para el uso de las redes sociales en la formación del profesional de la educación". Revista IPLAC, 4. (http://www.revista.iplac.rimed.cu/index. php?option=com_content\&view=category\&id=34\&I temid=270). (Recuperado el 21 abril de 2014).

Nápoles, N. (2011). Estrategia de Alfabetización Informacional para la producción de Objetos de Aprendizaje en la carrera de Ingeniería Agronómica en la Universidad Agraria de La Habana. Tesis en opción al Grado Científico de Doctor en Ciencias de la Educación. Universidad Agraria de La Habana.

Paradela, L. F. (2001) Una metodología para la gestión del conocimiento. Tesis Doctoral. Universidad Politécnica de Madrid.

Páez, M. y Díaz, T. (2012). "Modelo para la gestión del conocimiento pedagógico en la Universidad de Pinar del Río". Revista IPLAC, 4. (http://www.revista. iplac.rimed.cu/index.php?option=com_content\&vie $\mathrm{w}=$ category\&id=34\&Itemid=270) $($ Recuperado el 21 abril de 2014).

Prendes, M.P. (2011) "Innovación con TIC en enseñanza superior: descripción y resultados de experiencias en la Universidad de Murcia". Revista Electrónica Interuniversitaria de Formación del Profesorado, 14 (1); 267-280. (http//www.aufop.com) (Recuperado el 21 abril de 2014).

Roig, R. y Fiorucci, M. (2010). Claves para la investigación en innovación y calidad educativas. La integración de las Tecnologías de la Información y la Comunicación y la Interculturalidad en las aulas / Strumenti di ricerca per l'innovazione e la qualità in ambito educativo. Le Tecnologie dell'Informazione e della Comunicazione e l'Interculturalità nella scuola. 
Alcoy y Roma: Marfil y Università degli Studi Roma Tre.

Roig, R. y Flores, C. (2014). "Conocimiento tecnológico, pedagógico y disciplinario del profesorado: el caso de un centro educativo inteligente. EDUTEC Revista Electrónica de Tecnología Educativa, 41; 1-17. (http://edutec.rediris.es/Revelec2/Revelec47/n47 Roig-Flores.html) (Recuperado el 21 abril de 2014). Rosario, H. J. y Vásquez, L. F. (2012). "Formación del docente universitario en el uso de las TIC. Caso universidades públicas y privadas. (Universidades. de Carabobo y U. Metropolitana)". Pixel- Bit, 41; 163 171.

Torres, A. (2008) Una concepción teórico-metodológica para la producción de teleclases en formato digital destinadas a la Nueva Universidad Cubana. Tesis en opción al Grado Científico de Doctor en Ciencias de la Educación. Universidad Agraria de La Habana. 